\title{
La Competencia Cultural como referente de la Diversidad Humana en la Prestación de Servicios y la Intervención Social
}

The Cultural Competence and the Human
Diversity on the Social Intervention and the
Provision of Services

Manuel Francisco MARTÍNEZ1

Julia MARTÍNEZ ${ }^{2}$

Visitación CALZADO2

Fecha de Recepción: 18-09-2006

Fecha de Aceptación: 29-12-2006

\section{RESUMEN}

Los importantes cambios demográficos producidos en los últimos años en la sociedad española están teniendo un especial impacto sobre los servicios públicos: sanitarios, educativos, de bienestar, etc. El incremento de la diversidad cultural de los usuarios de estos servicios debe corresponderse con una mejora de la competencia cultural de sus profesionales para garantizar una atención pertinente y de calidad. En este trabajo se abordan las distintas dimensiones de la competencia cultural y su práctica en los distintos niveles de la intervención social.

\section{PALABRAS CLAVE}

Competencia cultural, Diversidad humana, Inmigración, Proveedores de servicios.

\footnotetext{
1 Universidad de Sevilla.

2 ADILA (Asociación de Intervención y Mediación Intercultural).
} 
La Competencia Cultural como referente de la Diversidad Humana en la Prestación de Servicios y la Intervención...

\section{ABSTRACT}

The important demographic changes that are taking place in the Spanish society have challenged services providers and organizations to consider cultural diversity as a priority. The purpose of this article is to present a model that will be helpful in providing culturally competent services. The concept of cultural competence is discussed and analyzed in the different levels of the social intervention.

\section{KEY WORDS}

Cultural competence, Human diversity, Immigration, Service providers. 
La tesis de que la dimensión cultural de los problemas sociales debe tenerse en cuenta en el diseño e implementación de las intervenciones comunitarias viene siendo defendida con fuerza en las últimas décadas (Bernal y Sáez-Santiago, 2006; Tricket, Watts y Birman, 1992; Marin y Marín, 1991; Sue y Zane, 1987). El principal sustento de dicha tesis está en el hecho de que si la conducta humana es aprendida y desplegada en un contexto cultural, toda intervención que quiera ser efectiva ha de prestar atención al entorno cultural donde están inmersos los individuos (Perdersen, 2003). Este enfoque ecológico-cultural de la diversidad humana permite contextualizar de manera más comprensiva y operativa los problemas de los miembros de los grupos minoritarios (inmigrantes, minorias étnicas, personas sin hogar, analfabetos, etc.) y explicar, a través de la teoría de la opresión (Nelson y Prilleltensky, 2005), el déficit estructural de recursos sociales y psicosociales que caracteriza a estos grupos humanos: ausencia o déficit de una imagen positiva de sí mismos, de participación en la agenda politica, de prestaciones sociales, etc.

En relación con la prestación de servicios (de bienestar, educativos, sanitarios, jurídicos, etc.) existe evidencia empírica de una baja utilización de los mismos en personas que tienen una mayor necesidad de ellos tales como minorias étnicas y/o emigradas (Bernal y Sáez-Santiago, 2006; Martínez \& Martínez, en prensa): latinos (Cherpitel, 2001; Power, et al., 2005), asiáticos (Zhang, et al., 1998), cubanos y puertorriqueños (Starret, et al., 1990), etc. Del mismo modo se han constatado dificultades en el proceso mismo de atención a los miembros de los grupos minoritarios como consecuencia de las diferencias culturales entre los actores, tal y como se recoge a modo de ejemplo (Saldaña, 2001) en la Tabla 1.
Estos problemas de accesibilidad a los recursos comunitarios y de adecuación de la atención recibida se han venido relacionando básicamente con las peculiares características (deficitarias, por supuesto) de los miembros de estos grupos minoritarios: escaso dominio del idioma, falta de hábitos adecuados de uso, búsqueda de otros recursos alternativos dentro de la comunidad étnica (sanadores, curanderos, entre otros), etc. Robinson \& Stalker (1993), por ejemplo, constataron menor accesibilidad a programas de respiro en minorias étnicas (inmigrantes en su mayoría) debido sobre todo al desconocimiento del idioma.

Sin embargo, desde el enfoque ecológico-cultural y la teoria de la opresión se explican los problemas de las minorias en general poniendo especial énfasis en las estrategias (conscientes o inconscientes) que sigue el grupo (o cultura) dominante para mantener su situación de privilegio respecto al disfrute de los recursos (Nelson \& Prilleltensky, 2005). De acuerdo con esta perspectiva, serian las características contextuales y organizacionales de los propios servicios (ausencia o escasa sensibilidad hacia las diversidad humana) los referentes de primer orden para explicar los problemas de accesibilidad y calidad de la atención recibida por esos grupos minoritarios: (1) falta de un ideario comprometido con la diversidad; (2) condiciones de desigualdad de poder en la relación profesionalusuario; (3) prácticas administrativas y atencionales no adecuadas a todo el rango que la diversidad humana implica; (4) profesionales escasamente concienciados o preparados para atender a los nuevos usuarios que configuran la realidad demográfica en la sociedad actual, etc. (Echeverry, 1997; Vázquez, 2005). Dado que la mayoría de los miembros de esos nuevos grupos minoritarios son subsidiarios de ayuda por su mayor vul- 
La Competencia Cultural como referente de la Diversidad Humana en la Prestación de Servicios y la Intervención...

Tabla. 1 Posibles errores en el proceso terapéutico en un contexto intercultural (Saldaña, 2001)

\begin{tabular}{|c|c|}
\hline Cliente/Usuario & Profesional/Terapeuta \\
\hline \multicolumn{2}{|c|}{ Compromiso } \\
\hline $\begin{array}{l}\text { 1. Se da cuenta de las diferencias } \\
\text { 2. Percibe distancia social } \\
\text { 3. Cree que el terapeuta no lo quiere } \\
\text { comprender } \\
\text { 4. Teme ser juzgado } \\
\text { 5. Manifiesta elevada ansiedad }\end{array}$ & $\begin{array}{l}\text { 1. Se dan cuenta de las diferencias } \\
\text { 2. Percibe distancia social } \\
\text { 3. Pautas stándards de comunicación } \\
\text { 4. Ve al usuario como estereotipo } \\
\text { 5. Manifiesta un aumento de la ansiedad }\end{array}$ \\
\hline \multicolumn{2}{|c|}{ Alianza terapéutica } \\
\hline $\begin{array}{l}\text { 1. No desarrolla un buen rapport } \\
\text { 2. Se siente incomprendido } \\
\text { 3. Manifiesta mucha desconfianza } \\
\text { 4. Disminuye su auto-revelación }\end{array}$ & $\begin{array}{l}\text { 1. Cree que el usuario es resistente } \\
\text { 2. No comprende al usuario } \\
\text { 3. No responde adecuadamente a la } \\
\text { desconfianza } \\
\text { 4. Ve al usuario sin motivación o } \\
\text { psicológicamente no mentalizado }\end{array}$ \\
\hline \multicolumn{2}{|c|}{ Resultados } \\
\hline $\begin{array}{l}\text { 1. Muestra frustración/ ansiedad } \\
\text { 2. Cancela las sesiones } \\
\text { 3. Falla en las citas } \\
\text { 4. Abandona prematuramente el } \\
\text { tratamiento }\end{array}$ & $\begin{array}{l}\text { 1. Muestra ansiedad y frustración } \\
\text { 2. Manifiesta mala alianza; puede hacer } \\
\text { un mal diagnóstico } \\
\text { 3. Percibe una planificación defectuosa del } \\
\text { tratamiento } \\
\text { 4. Observa resultados erróneos }\end{array}$ \\
\hline
\end{tabular}

nerabilidad, el desarrollo de intervenciones sensibles a la diversidad humana se ha convertido en uno de los principales retos a los que tienen que enfrentarse los servicios públicos y los profesionales de la intervención social en general.

Consciente de estos hechos y desde varias disciplinas aplicadas (sanitarias, educativas, de protección social, etc.) se vienen acuñando diversos constructos teóricos y modelos aplicados que tienen en común el destacar la necesidad de cambios sociales profundos (en lo personal, institucional y funcional) que posibiliten una prestación de servicios de calidad y apropiada al conjunto de los usuarios pertenecientes a los grupos minoritarios. Aunque no exento de polémica, uno de los constructos que ha tenido una mayor difusión, y que se está demostrando útil en la mejora de la prestación de servicios y la intervención social, es el de competencia cultural, a cuya naturaleza teórica y aplicada se referirán los siguientes apartados.

\section{SOBRE EL CONCEPTO DE COMPETENCIA CULTURAL}

En sentido amplio la cultura puede ser entendida como el patrón integrado de conductas humanas -pensamientos, comunicación, prácticas, costumbres, creencias, valores, instituciones, etc.- de grupos específicos definidos por dimensiones como la etnia, género, nacionalidad, status socioeconómico, opción sexual, religiosa o política, etc. Por su parte, la competencia se refiere, con carácter general, a la habilidad para funcionar eficazmente. En consecuencia, se puede definir competencia cultural como el conjunto de conocimientos, actitudes, conductas, y en su caso políticas y programas, que confluyen en una persona, organismo o sistema que le capacitan para trabajar (convivir, ser implementado, etc.) con eficacia en contextos interculturales. Por tanto, la competencia cultural puede estar referida a ciudadanos, profesionales de los servicios, politicos, a un barrio, centro de salud o de 
servicios sociales, o al sistema jurídico, educativo, etc.

La competencia cultural es un constructo complejo y no sólo por las múltiples dimensiones que la configuran, sino también porque es al mismo tiempo un proceso y un resultado. Hayes (1991) la concibe con una estructura dinámica que se mueve en un eje en el que representa los continuos progresos (avances) que realizan las personas (instituciones, sistemas, etc.) hasta alcanzar el grado óptimo de funcionamiento en contextos culturales (Figura 1). De acuerdo con este autor, la adquisición de una competencia cultural eficaz es el resultado de un proceso de desarrollo de capacidad que no sigue un modelo lineal. Cada persona (sistema, institución, programa, etc.) progresa con un ritmo y trayectoria determinada, afrontando sus propios contratiempos y logrando mejoras específicas en cada una de las etapas. Además, los distintos ámbitos que abarca el logro competencial cultural (género, etnia, orientación sexual, etc.) suelen seguir patrones diferentes de logro (en tiempo e intensidad) en un sujeto dado. De esta forma, es frecuente encontrar personas (instituciones, sistema, etc.) que alcanzan una alta competencia cultural en relación a la perspectiva de género, por ejemplo, y se mantienen incapaces en otros contextos: interétnico, político, religioso, etc.
Como proceso, y desde el ámbito de la intervención social, la competencia cultural profesional supone la continua mejora en el reconocimiento de las dimensiones culturales del trabajo (clínico, social, educativo, etc.). Ello incluye: (1) la aceptación de las diferencias intergrupales en la forma de ver el mundo, los problemas sociales, la salud, los efectos del racismo y la opresión, etc.; (2) la capacidad para tratar a las personas (usuarios, alumnos, pacientes, etc.) con flexibilidad y sensibilidad cultural; y (3) la capacidad para entender las propias ideas, predisposiciones y reacciones. En consecuencia, la competencia cultural implica las siguientes dimensiones (Campinha-Bacote, 1998; Sue, Arredondo y McDavis, 1992):

1) conciencia de las propias actitudes y creencias,

2) conocimiento acerca de las diferencias culturales entre los diversos grupos en que se manifiesta la diversidad humana,

3) habilidades para trabajar con esos grupos diversos,

4) la necesidad de una efectiva interacción con los miembros de otros grupos en específicos encuentros culturales.

Además de estas cuatro dimensiones,

Figura 1. La competencia cultural como continuum (Hayes, 1991)

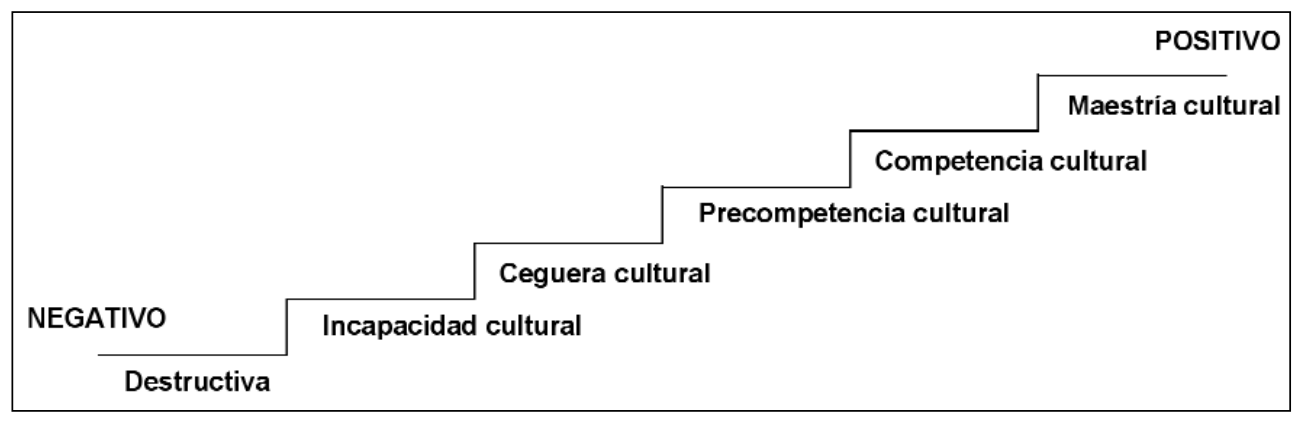


Campinha-Bacote (2002) cree que también es importante tener en cuenta el deseo cultural, es decir, el grado de la motivación de la persona (o del profesional) para comprometerse en el proceso de adquisición de conciencia, conocimientos y habilidades culturalmente adecuadas para trabajar con grupos humanos de diversos background cultural. De acuerdo con la autora, este deseo debe ser genuino y auténtico, fruto de una aspiración del profesional y no sólo de haber asumido la obligación de tener que implicarse en ese proceso de cambio.

El conjunto de estos elementos se configuran en un modelo dinámico según el cual el proceso de adquisición de competencia cultural resulta de la intersección de todos ellos (Figura 2). De acuerdo con este modelo, y en relación con la atención a grupos minoritarios de distinto background cultural, una autoevalución de la competencia cultural de los profesionales de la intervención social supone contestar a preguntas cla- ves como: (1) ¿Soy consciente de mis sesgos personales y prejuicios hacia grupos culturales diferentes del mío?; (2) ¿Tengo las habilidades necesarias para realizar una evaluación cultural y diseñar un plan de acción culturalmente sensible?; (3) ¿Tengo conocimientos significativos de los elementos más importantes de la cultural del usuario y de la diversidad humana en general?; (4) ¿Cuántos encuentros cara a cara he tenido con usuarios de distinto backgroud cultural?; y (5) ¿Cómo es de genuino mi deseo de querer ser culturalmente competente?

La competencia cultural puede convertirse en el futuro en el constructo de mayor relieve para lograr una prestación de servicios sensible a la diversidad. Sin embargo, para alcanzar este objetivo debe tener un mayor desarrollo desde esta concepción comprensiva que acabamos de exponer hacia una verdadera y efectiva práctica. En este sentido, Sue (2006) ha planteado recientemente algu-

Figura 2. Dimensiones de la competencia cultural (Campinha-Bacote, 1998)

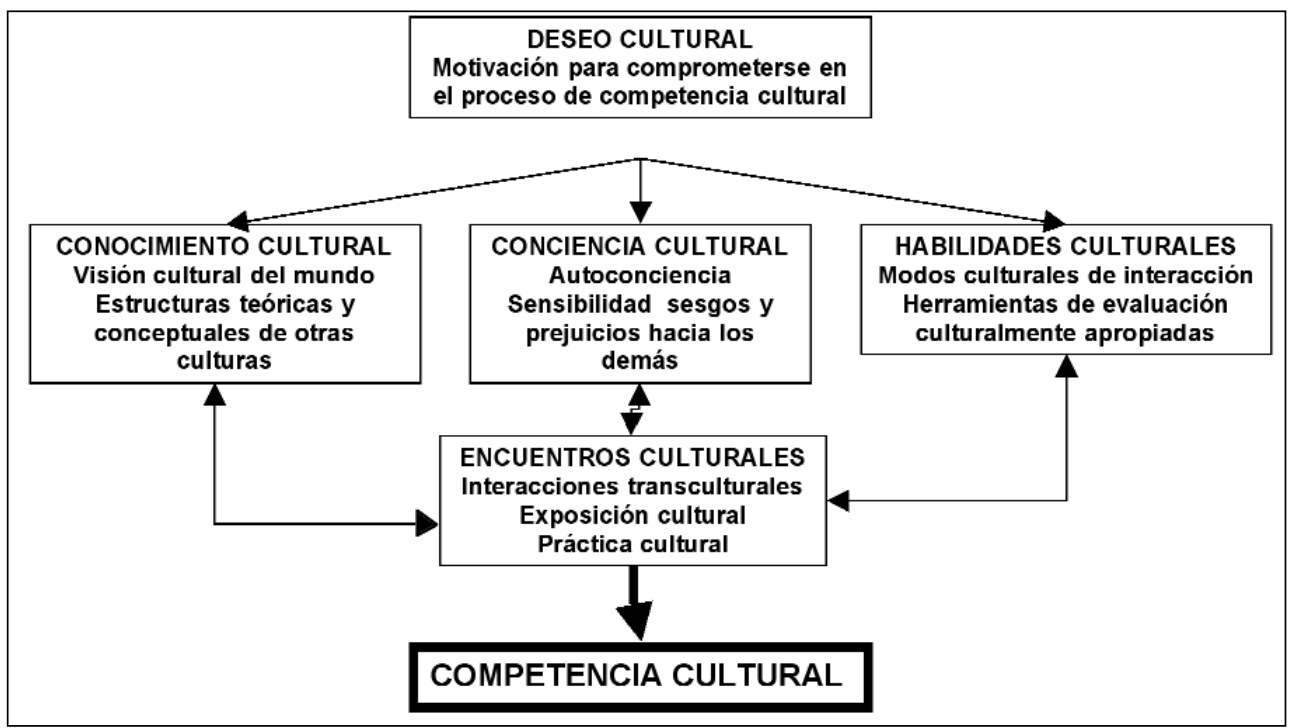


nos interrogantes que representan un reto para profesionales e investigadores para los próximos años: (1) Si la competencia cultural implica conocimiento, ¿es posible conocer todas las culturas?, ¿Cuánto y qué conocimiento cultural es el necesario?; (2) ¿Existen diferentes competencias en función de los grupos minoritarios de referencia?, o ¿la competencia cultural reside en el individuo (profesional) independientemente de dichos grupos?; (3) Si la competencia cultural es un constructo multidimensional, ¿son todas sus dimensiones igual de importantes?. Para ayudar a contestar a estas preguntas en relación a la prestación de servicios, en los siguientes apartados se profundizará en los distintos componentes de la competencia cultural.

\section{CONCIENCIA CULTURAL.}

Como ocurre en cualquier persona, el profesional de la intervención social se ve influenciado en la conformación de su visión del mundo por su propio contexto cultural de pertenencia (Pedersen, 2000; Sue, Arredondo y McDavis, 1992). Por ello, tiene que aprender a reconocer que esa pertenencia a grupos (culturales) especificos puede conducirle a adoptar actitudes $\mathrm{y} / \mathrm{o}$ creencias que tengan una influencia negativa sobre su manera de interactuar con individuos (usuarios, clientes, compañeros, etc.) que pertenecen a grupos culturalmente distintos (por nacionalidad, etnia, religión, género, etc.). Si en la prestación de servicios están implicadas la manera de percibir al usuario, sus problemas y la relación profesional que con él se establece (Sue, Ivey y Pedersen, 1996), la conciencia cultural será el proceso a través del cual el profesional llega a respetar, apreciar y ser sensible hacia los valores, creencias, estilos de vida, prácticas, estrategias para resolver problemas, etc. de la cultura del usuario.
Este proceso implica un continuo examen de los propios sesgos y prejuicios hacia otras culturas, así como una exploración en profundidad del propio background cultural. Sin ser consciente de los valores de nuestra propia cultura y sus implicaciones prácticas, corremos el riesgo practicar una imposición cultural (Leininger, 1978). En este sentido se ha señalado que en la psicoterapia tradicional subyacen una serie de conflictos relacionados con ciertos valores sostenidos por algunos grupos minoritarios (Nagayama-Hall, 2001). Así, por ejemplo, el tratamiento psicológico convencional tiende a promover los valores propios de la cultura individualista: autonomía individual, competitividad, limites nítidos entre el self y los otros, emociones ego centradas, asertividad, locus of control interno, etc.; por el contrario, algunos grupos socializan a sus miembros en los valores propios de la cultura colectivista: armonía con el grupo, cooperación, self interdependiente, conexión con los valores grupales; valoración del logro grupal, fomento de la equidad, etc. El problema puede surgir no tanto de la preferencia que tenga el profesional hacia ciertas personas, orientaciones sociales, modelos, valores etc., sino que evalúe como negativas otras preferencias y actúe en consecuencia.

Desde la Psicologia Social se ha puesto de manifiesto que las percepciones que tenemos de los miembros de los otros grupos, y del mundo en general, se conforman y se estructuran a través del proceso psicológico de la categorización social (Tajfel y Turner, 1986, Fiske, 1998). A través de ella se asocian rasgos y comportamientos a grupos determinados siguiendo una serie de leyes o normas tales como: (1) Los individuos perciben más homogéneos a los miembros de su propio (endo) grupo y exageran las diferencias respecto de los miembros de los demás (exo) grupos; y (2) Esta distin- 
ción conlleva al mismo tiempo un cierto favoritismo hacia los miembros del endogrupo (sesgo endogrupal). Esta forma de proceder se hace de forma inconsciente y puede resultar problemática (y conducir al prejuicio, discriminación, exclusión, etc.) cuando un grupo tiene más poder que otro (profesionales vs usuarios, por ejemplo), o cuando los recursos que hay en un determinado contexto no están repartidos equitativamente (población autóctona vs inmigrante). Por ello, se debe aceptar que la interpretación que hace el profesional de algunas conductas de las personas (relacionadas con la salud/enfermedad, por ejemplo) pueda ser inconscientemente prejuiciosa y esté influenciada por estereotipos negativos, elementos constitutivos del proceso de categorización social.

La conciencia cultural debe ser un elemento crítico en el proceso de actualización formativa de los profesionales de los servicios sociales, de salud, educativos, etc. y les debe conducir desde el etnocentrismo a un etnorelativismo cultural, sin que esto signifique que han de ser aceptadas todas las prácticas culturales. Como ya se ha indicado, implica la adquisición de conocimientos (valores, creencias, patrones de conducta, etc.) acerca de sus propios entornos culturales (social us politico, oprimido vs privilegiado, etc.) que han influido sus actitudes, estereotipos, ideas preconcebidas, comportamiento, etc. Al tratarse de cuestiones especialmente delicadas, y en la que los profesionales pueden sentirse vulnerables, los procesos de aprendizaje en este área deben hacerse en un clima abierto, positivo y de total confianza.

En la literatura especializada se han descrito diversas estrategias para amortiguar (y/o reducir) el sesgo endogrupal y mejorar de esta forma las relaciones intergrupales. Una de ellas se refiere a la adopción de una perspectiva daltónica al interaccionar con personas de otra etnia, género, etc. minimizando las diferencias (politicas, étnicas, etc.) y poniendo el énfasis en los aspectos universales del comportamiento (APA, 2003). De esta forma se puede disminuir la desigualdad al minimizar el pensamiento categórico, el uso de estereotipos, la preferencia por el endogrupo, etc. Sin embargo, los datos de la investigación en el área no son del todo concluyentes y el ignorar las diferencias integrupales puede conducir, como efecto no deseado, a un status quo en el que no necesariamente se produce un tratamiento equitativo entre grupo mayoritario y minoritario (Schofield, 1986; Wolsko, Park, Judd y Wittenbrink, 2000).

Siguiendo las recomendaciones de la American Psychological Association, para reducir los estereotipos y prejuicios de los profesionales de la intervención social (APA,2003), se pueden utilizar las siguientes estrategias: (1) mejorar el conocimiento de las propias creencias, valores, actitudes etc.; (2) esforzarse y ejercitarse para cambiar las percepciones automáticas positivas para el endogrupo y negativas para el exogrupo; (3) percibir a las personas como individuos y no como miembros de un grupo; (4) cambiar la percepción de nosotros vs ellos por la de nosotros; y (5) recategorizar a los miembros del exogrupo como miembros del endogrupo. Estos modelos son útiles sólo cuando el profesional se esfuerza por ser más abierto y trabaja para mejorar la comunicación interpersonal.

\section{CONOCIMIENTO CULTURAL}

Uno de los requisitos más importantes para comprender la conducta del usuario, paciente o vecino es conocer su forma de ver el mundo. Por ello es importante buscar y adquirir un sólido 
conocimiento cultural y comprensión acerca de las diferencias étnicas de los individuos. Este conocimiento multicultural conlleva un aprendizaje y búsqueda de información sobre culturas, cosmovisiones y experiencias de los diferentes grupos de personas: patrimonio, historia, estructura familiar, valores, creencias, etc. En el campo de la salud mental, por ejemplo, es importante para realizar un adecuado diagnóstico y tratamiento conocer del usuario sus creencias sobre el origen y naturaleza de la salud mental, la forma de manifestarse los sintomas, el estilo de afrontamiento, el sistema de apoyo familiar y comunitario, la disposición para el cumplimiento del tratamiento, etc. Asimismo, se debe tener información precisa sobre las barreras institucionales, el contexto sociopolítico y los mecanismos de opresión y discriminación que sufre el grupo minoritario en cuestión (Sodowsky, KuoJackson, \& Loya, 1997). Esta dimensión de la competencia cultural debe completarse con una actitud de búsqueda continua de información y de formulación de preguntas apropiadas y significativas.

Purnell \& Paulanka (1998) distinguen cuatro niveles de conocimiento cultural: (1) Incompetencia inconsciente, o sindrome de la ceguera cultural, cuando el profesional no es conciente de su carencia de conocimiento cultural; (2) Incompetencia consciente, cuando se conoce la importancia de la cultura en la prestación de servicios a poblaciones diversas (a través de cursos, textos, o encuentros culturales profesionales) pero no se tienen los conocimientos necesarios; (3) competencia consciente es el acto intencionado de aprender acerca de la cultura de los usuarios ofreciendo respuestas profesionales culturalmente sensibles; (4) Competencia inconsciente es la habilidad automática para ofrecer servicios culturalmente congruentes a las características de los usuarios.
A modo de orientación se recogen a continuación algunos de los conocimientos más relevantes que se deben adquirir para alcanzar la competencia cultural profesional:

1. Cultura de los usuarios: (historia, tradiciones, valores, sistema familiar, expresiones artísticas, etc.

2. Impacto del racismo y la pobreza sobre la conducta, actitudes, valores y discapacidades.

3. Patrones de conductas de búsqueda de ayuda de los usuarios de los grupos minoritarios.

4. Papel del lenguaje, patrones en la forma de hablar y estilos de comunicación en las diferentes comunidades.

5. Impacto de las politicas de servicios públicos sobre los usuarios de los distintos grupos minoritarios.

6. Recursos (centros, personas, redes de apoyo informal, etc.) disponibles para las grupos minoritarios y las comunidades.

7. Reconocer cómo los valores de los profesionales puede entrar en conflicto 0 acomodarse a las necesidades de los usuarios de diferentes culturas.

8. Conocer cómo las relaciones de poder en las comunidades o en las instituciones impacta en las diferentes culturas.

9. Etc.

\section{HABILIDADES CULTURALES}

Esta tercera dimensión se refiere a la ejecución de prácticas profesionales culturalmente apropiadas a las característi- 
cas de los usuarios con los que se interactúa. Estas prácticas van desde el uso apropiado del lenguaje, la forma de desarrollar la entrevista, los datos a obtener del usuario, la elaboración del diagnóstico o el diseño de un programa de intervención. El conocimiento y la conciencia cultural abordados en párrafos anteriores deben ser empleados para desarrollar las habilidades multiculturales, es decir, comportamientos e intervenciones apropiadas y culturalmente sensibles. La vía hacia el desarrollo de estas habilidades es la propia exploración y conciencia cultural, y conforme los profesionales sean más conscientes de sus propias ideas, valores, concepciones y estereotipos, tenderán a proyectar en menor medida sus propios valores culturales a los usuarios, por lo que se volverán más eficientes (Sue, Ivey y Pederson, 1996).

De acuerdo con el efecto de primacía (Asch, 1946), los primeros elementos informativos que recibe el perceptor son muy importantes ya que se constituyen en un filtro que organiza y estructura la información posterior. Por ello, es importante la habilidad del profesional para establecer desde el principio una relación positiva de comunicación con el usuario (y en su caso la familia), creando un clima cálido, respetuoso y de confianza. Para ello debe, si es posible, conocer y pronunciar correctamente su nombre, mantener una distancia personal apropiada a su cultura, cuidar las formulas de cortesia y el contacto visual (en algunas culturas es improcedente mirar directamente a los ojos al interlocutor), etc. Igualmente hay que destacar las habilidades necesarias para realizar una adecuada evaluación diagnóstica del usuario, paciente, trabajador, etc. El lenguaje, como vehículo de expresión de emociones y sentimientos ocupa, en este ámbito, un lugar central por lo que la utilización de herramientas de evaluación culturalmente apropiadas (traduc- ciones culturalmente adaptadas, por ejemplo) se hace fundamental para conocer de forma fiable el funcionamiento psicológico del individuo. En esta misma linea, es importante la habilidad del profesional para elicitar en el usuario la forma de entender su enfermedad (o problema), sus creencias sobre la causa de la misma, el tipo de tratamiento que le parece más adecuado, la forma de afrontar el dolor, el papel de la familia en la resolución del problema, etc.

Como en los apartados anteriores se ofrece a continuación un listado de otras habilidades culturalmente apropiadas en la prestación de servicios:

1. Técnicas para aprender las culturas de los grupos minoritarios.

2. Habilidades para comunicar información precisa sobre los usuarios miembros de esos grupos.

3. Habilidad para discutir abiertamente sobre temas/diferencias étnicas, y responder con ejemplos basados en la cultura.

4. Habilidad para evaluar el significado que la etnicidad tiene para los usuarios.

5. Habilidad para discernir entre los sintomas (problemas) que tienen su causa en el propio usuario y los que se derivan de la estructura social.

6. Técnicas de entrevistas que ayuden al profesional a comprender y acomodar el lenguaje a la cultura del usuario.

7. Habilidad para utilizar el concepto de empowerment a favor de las los miembros de los grupos minoritarios y sus comunidades.

8. Habilidad para usar los recursos a 
favor de los usuarios de los grupos minoritarios y sus comunidades.

9. Habilidades para reconocer y combatir el racismo, estereotipos étnicos y mitos entre los individuos e instituciones.

10. Habilidades para desarrollar y validar nuevas técnicas de investigación y evaluación aplicadas al trabajo con los grupos minoritarios.

\section{ENCUENTRO CULTURAL}

Es el proceso que estimula al profesional a comprometerse directamente en interacciones transculturales con usuarios de diversos background (CampinhaBacote, 2003). Debido a que la variabilidad endogrupal es mayor que la intergrupal, para que estos encuentros sean significativos han de realizarse con el mayor número de sujetos y garantizar un conocimiento suficiente de los valores, creencias y prácticas específicas del grupo en cuestión. En Psicologia Social existe una amplia tradición en el estudio de los encuentros intergrupales a partir de la llamada hipótesis del contacto. De acuerdo con ella, las características sociales, situacionales y personales en las que se produce el contacto determinan el resultado del mismo: (1) intensifica el prejuicio fomentando el desarrollo de antipatía personal y colectiva hacia los "otros"; o (2) puede reducir el prejuicio cuestionando la validez de las actitudes negativas, la ansiedad y los estereotipos.

En los contactos que se establecen entre el profesional y los usuarios es necesario cuidar al máximo las características en las que éste se produce para obtener un resultado positivo que permita una atención pertinente y de calidad. Aunque en la Tabla 2 se describen los factores más significativos que modulan el resultado del contacto cultural entre grupos, algunos son especialmente relevante en la relación que se establece entre el profesional de los servicios y los usuarios.

Siguiendo a Sue et al., (1992), los encuentros cara a cara permiten: (1) redefinir la idea que se tiene de ese grupo; (2) ayuda a prevenir y/o desmon-

Tabla 2. Factores determinantes del resultado del contacto cultural

\begin{tabular}{|l|}
\hline Factores Sociales: \\
\hline - Estructura social \\
- Grado en que la sociedad esta organizada en jerarquías basadas en el poder, \\
etnia, género, religión, etc. \\
- Historia de las relaciones entre los grupos que entran en contacto \\
- Relaciones actuales de los grupos \\
- Background cultural de los individuos que entran en contacto \\
\hline Factores Situacionales \\
- El escenario en que tiene lugar el contacto \\
- La naturaleza de la interacción \\
- El tipo de tareas a ejecutar \\
Factores Personales \\
- Caracteristicas sociodemográficas de los individuos \\
- Rasgos de personalidad \\
\hline Factoritudes y creencias que tienen de la situación de contacto \\
contacto, y los resultados del mismo \\
- Respuestas cognitivas de los intervinientes \\
- Respuestas afectivas de los intervinientes \\
\hline
\end{tabular}


tar los falsos estereotipos; (3) generar una amplia gama de respuestas verbales; y; (4) enviar y recibir mensajes verbales y no verbales apropiados y precisos. Especial importancia adquiere en estos encuentros el contacto visual, las expresiones faciales, el contacto corporal, el lenguaje del cuerpo, la distancia, etc.

Los encuentros culturales también incluyen una valoración de las necesidades lingüisticas del usuario y del profesional. Usar un intérprete adecuadamente entrenado (o un servicio de traducción como el que está ofreciendo actualmente en el Servicio Andaluz de Salud) facilita, en su caso, la comunicación precisa durante los encuentros y es una respuesta que en muchos casos puede ser suficiente para una comunicación efectiva. El uso de intérpretes inexpertos como amigos o familiares puede ser otra alternativa a valorar, teniendo en cuenta que la falta de conocimientos de estas personas sobre el problema en cuestión (sanitario, jurídico, social, etc.) puede acarrear errores importantes tanto en el diagnóstico como en el plan de acción.

\section{LA COMPETENCIA CULTURAL EN EL NIVEL INSTITUCIONAL}

En la prestación de servicios, la competencia cultural no sólo se refiere a los profesionales que realizan la atención al usuario sino que también ha de tenerse en cuenta la dimensión organizacional desde donde se presta el mismo (escuela, centro de servicios sociales, centro de salud, etc.). De acuerdo con Cross, Bazron, Dennos e Isaacs (1989), en el nivel institucional la competencia cultural debe regirse por una serie de parámetros definitorios para una adecuada gestión de la diversidad: (1) valorar la diversidad, es decir valorar la forma en que los usuarios definen salud, bienestar, educación, etc.; (2) tener la capacidad de autoevaluarse culturalmente; (3) ser consciente de la dinámica inherente a la interacción entre las culturas; (4) contar con conocimientos culturales institucionalizados; y (5) haber adaptado la prestación de los servicios para que refleje una comprensión del pluralismo cultural.

En la práctica se trataría de que la organización (comprometiendo al mayor número de sus miembros) declare su voluntad de trabajar por mejorar la competencia de sus servicios en relación a la diversidad cultural. Esta declaración puede efectuarla en un manifiesto concreto y/o incorporarla en la misión o ideario del centro. Debe también realizar una autoevaluación inicial que le permita definir, para un tiempo dado, objetivos, acciones y recursos que comprometerá para lograr aquella mejora. Por último, diseñará un plan de evaluación que valore los objetivos alcanzados y oriente la planificación de nuevas acciones para el siguiente periodo. Ello se concreta en un plan de acción estratégico, integral y coordinado, que debe plasmarse en todos los niveles de la organización: (1) formulación de politicas; (2) creación de una adecuada infraestructura; (3) administración y evaluación de los servicios, programas, etc.; (4) prestación de servicios y mecanismos que la sustenten; y (5) nivel personal referido tanto a profesionales como usuarios.

En todo este proceso de ganancia de competencia cultural organizacional juega un importante papel la autoevaluación (Leininger, 1978). La evaluación debe cubrir diversos ámbitos organizacionales, tales como recursos humanos, prácticas atencionales, recursos materiales (protocolos, folletos, materiales educativos, etc.), entorno físico, politica de personal, programas de intervención, etc. En las Tablas $3 a, 3 b$ y $3 c$ se recogen algunos items (Sutton, 2000) que pueden incorpo- 
rarse a un cuestionario de autoevaluación de los profesionales de un servicio en relación a su competencia cultural para atender a grupos minoritarios. Los sujetos deben responder A (Frecuentemente), $\mathrm{B}$ (Ocasionalmente) o C (Raras veces 0 nunca) de acuerdo con la frecuencia con que ejecutan las acciones que se describen en cada uno de los items.

Aunque en España el paradigma de la competencia cultural no está aun muy difundido en la prestación de servicios, existen instituciones y/o centros de atención (sanitaria, educativa, de servicios sociales, de recepción a inmigrantes, etc.) que han implementado planes de formación y entrenamiento en diversidad cultural para sus profesionales y han elaborado diverso material con información relevante en los idiomas más representativos de su comunidad de usuarios. Se hecha en falta material divulgativo dirigido a los profesionales de atención directa con información significativa y útil en relación a los distintos grupos culturales a los que tienen que atender. En el caso de los inmigrantes, estas guías breves podrian contener información sobre: (1) Características generales de la migración del grupo en cuestión: latinoamericanos, subsaharianos, chinos, etc.; (2) Creencias sobre la salud, el bienestar y las necesidades sociales; (3) Patrones de conducta de búsqueda de ayuda y utilización de servicios; (3) Familia, infancia y tercera edad; (4) Elementos clave para una comunicación eficaz: (forma de presentarse, utilización del lenguaje no verbal, proxemia, giros lingüísticos significativos, etc.; (5) Principales necesidades sociales, de bienestar y salud; etc.

Algunas asociaciones profesionales han constituido grupos específicos de trabajo para diseñar estrategias y directrices prácticas que ayuden a incorporar los temas de diversidad en la formación, entrenamiento, investigación, práctica, etc. de sus profesionales. Trimble, Stevenson y Worell (2003) plantean que una práctica verdaderamente inclusiva por

\section{Tabla 3a. Cuestiones relevantes en la evaluación cultural de un servicio (Modificado de Sutton, 2000)}

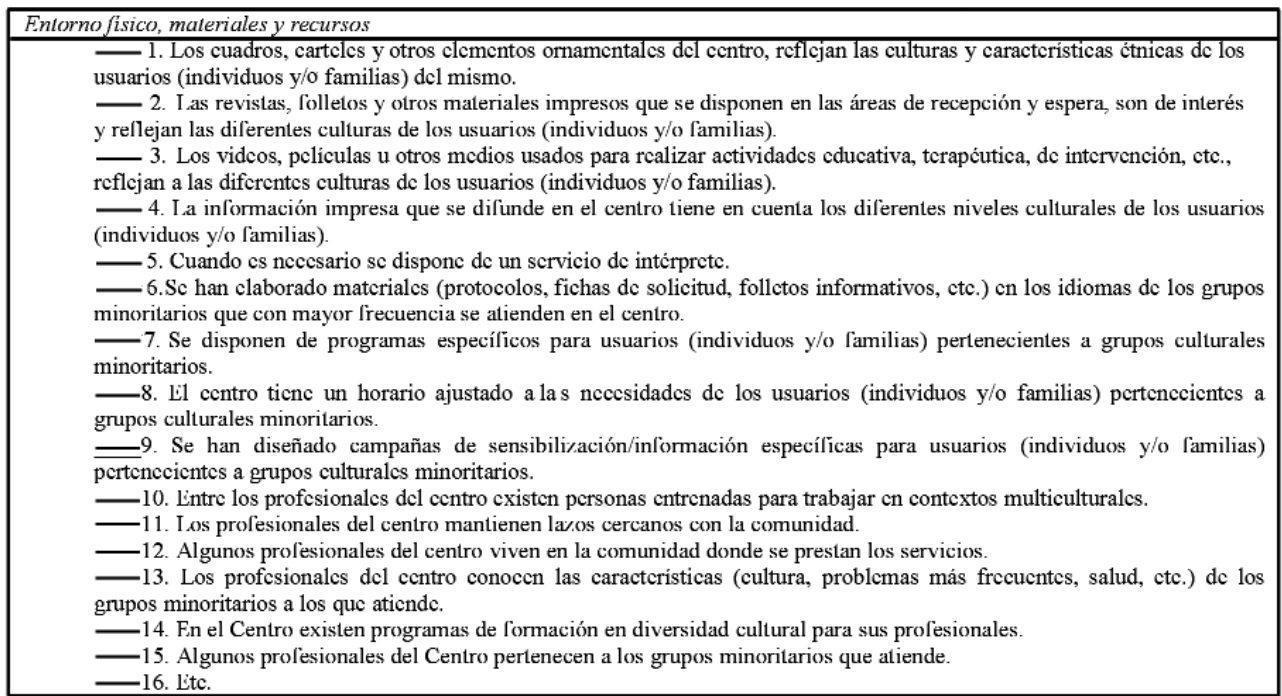


La Competencia Cultural como referente de la Diversidad Humana en la Prestación de Servicios y la Intervención...

\section{Tabla 3b. Cuestiones relevantes en la evaluación cultural de un servicio} (Modificado de Sutton, 2000)

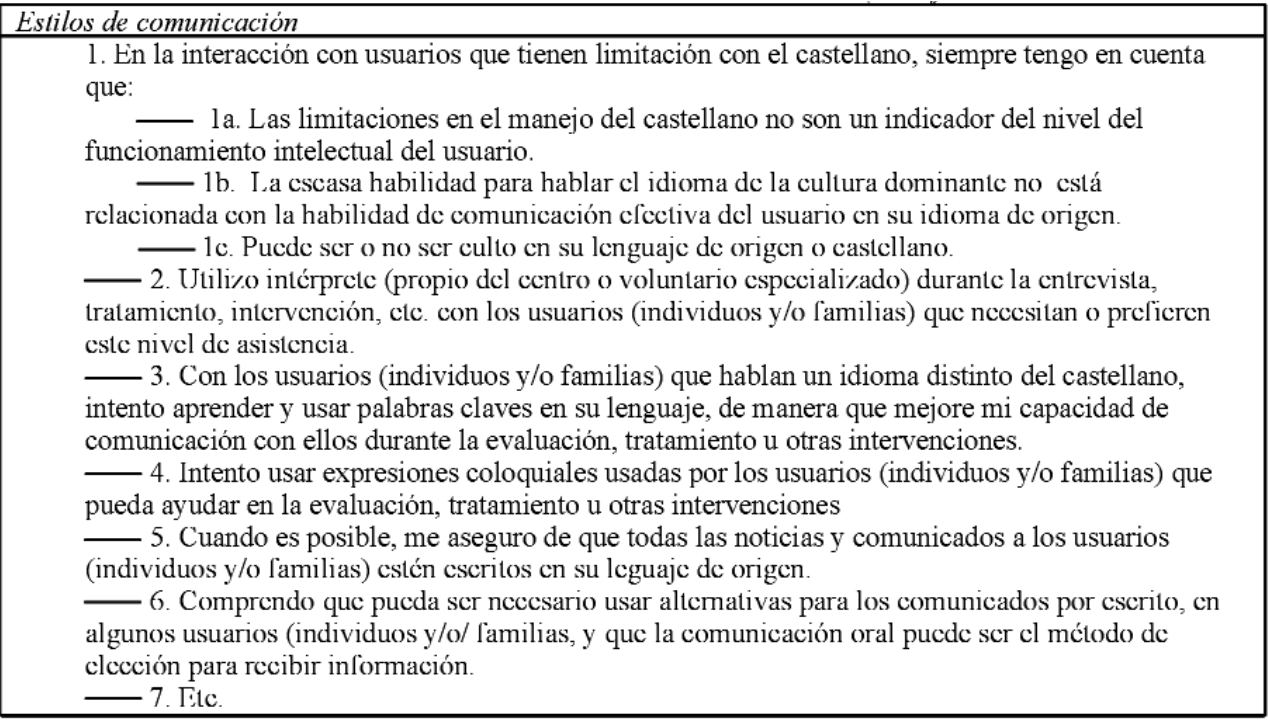

parte de los profesionales de la intervención social debe regirse por una serie de principios relacionados con el uso del lenguaje -que refleja constructos cultural e ideológicamente determinados-, con resaltar las fortalezas de los grupos minoritarios más que su rasgos diferenciales o déficits, con la simplificación de la realidad a través del usos de tests, cuestionarios, etc. porque aísla a la persona de su contexto social., etc. La guía sobre tratamientos psicológicos para minorías étnicas promovida por el Consejo Nacional de Asociaciones Psicológicas para el Avance de los Intereses de las Minorías Étnicas (APA, 2003), es una buena muestra de adaptación cultural de la intervención psicológica dirigida a población latina, negra, india y asiática.

En cuanto a los programas de intervención diseñados desde las organizaciones, la competencia cultural se refiere a la capacidad de los mismos para respetar y valorar las creencias, los estilos interpersonales, las actitudes y las conductas de la población diana y los profesionales implicados en el mismo (Roberts et al., 1990). El diseño de programas de intervención culturalmente sensibles está siempre justificado por los siguientes factores: (1) Muestra el respeto hacia los valores de la cultura de la comunidad; (2) Mejora nuestra habilidad para conectar con los miembros de la comunidad; (3) Aumenta la relevancia de nuestras acciones y disminuye la posibilidad de sorpresas no deseadas; (4) Aumenta la inclusión y la participación en el programa de los miembros de la comunidad; y el apoyo de aquellos que no directamente involucrados en el mismo; (5) Aumenta la probabilidad de éxito e impacto de nuestra intervención; y (6) Incrementa la confianza y la cooperación en el ámbito cultural, y se generan mejores perspectivas para intervenciones exitosas en el futuro. En la Tabla 4 se describe, a modo de ejemplo, una actuación sencilla realizada por Sandra Michie, 


\section{Tabla 3c. Cuestiones relevantes en la evaluación cultural de un servicio} (Modificado de Sutton, 2000)

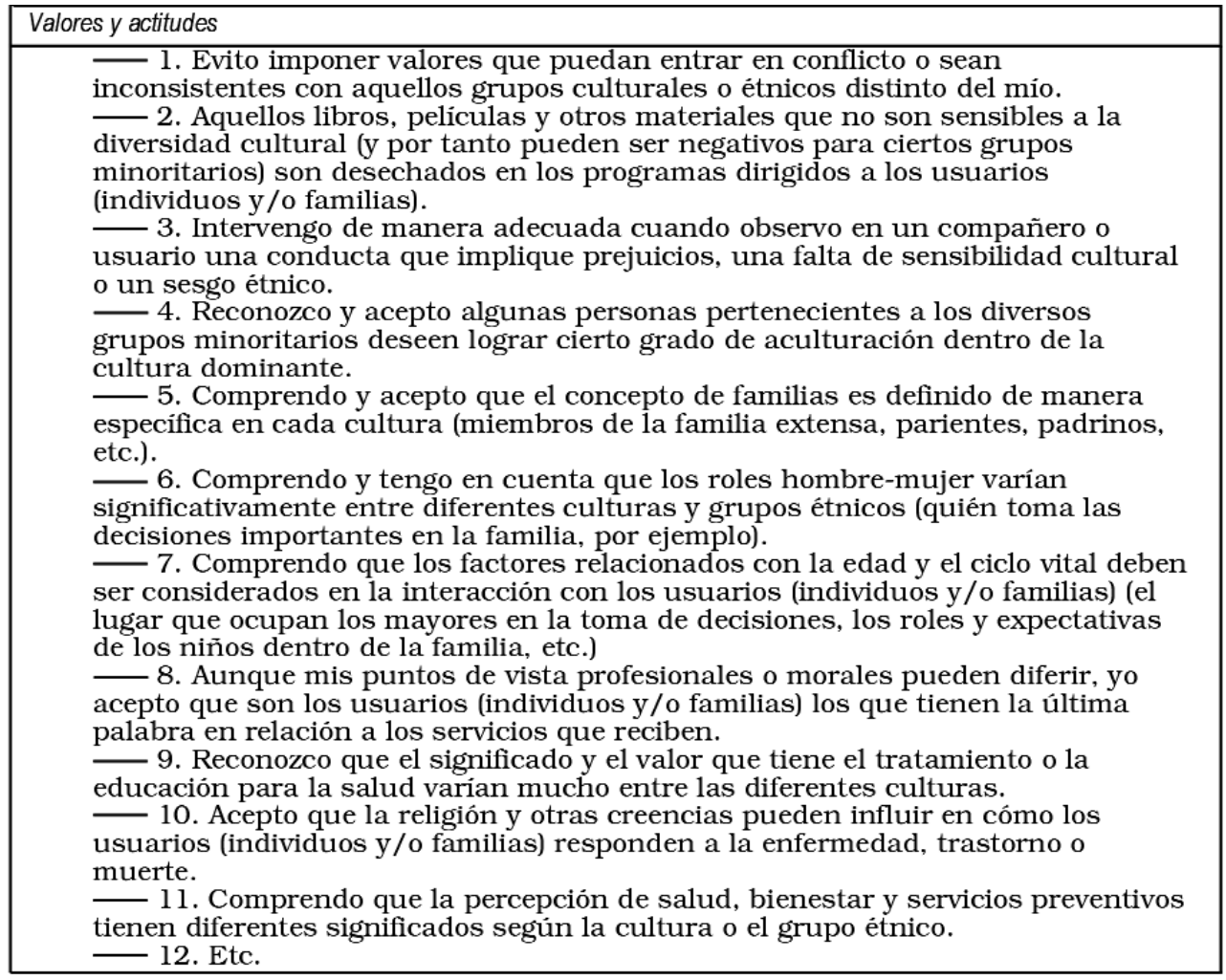

profesional de la salud que pasó 25 años en Zambia En esta intervención tuvieron que tenerse en cuenta los puntos de vista e intereses contrapuestos de los grupos implicados: personas mayores vs jóvenes; con educación sanitaria vs analfabetos; varones vs mujeres.

Por último, hay resaltar en este apartado la importancia que tiene la promoción de la colaboración entre los servicios formales suministrados por la administración pública y los que prestan desde el mismo contexto comunitario. En España, y en el ámbito de la inmigración por ejemplo, la iniciativa social (ONGs, empresas sociales, fundaciones, etc.) viene desplegando una amplia y eficiente carta de servicios culturalmente sensibles que tienen como referente teórico y metodológico la mediación intercultural. Esta importante atención directa que se realiza desde las organizaciones sociales (a una población muy vulnerable y en contextos en los que difícilmente llegan los servicios formales), debería complementarse con otras actividades (protocolizadas) de colaboración con los centros públicos (educativos, sanitarios, sociales, etc.) dirigidas a prestar servicios de interpretación/traducción, de mediación intercultural en caso de conflicto, intermediación, de acompañamiento $\mathrm{y} / 0$ seguimiento, etc. 
La Competencia Cultural como referente de la Diversidad Humana en la Prestación de Servicios y la Intervención...

Tabla 4. Descripción de un caso de intervención culturalmente adaptada (descrito por Michie, 2006)

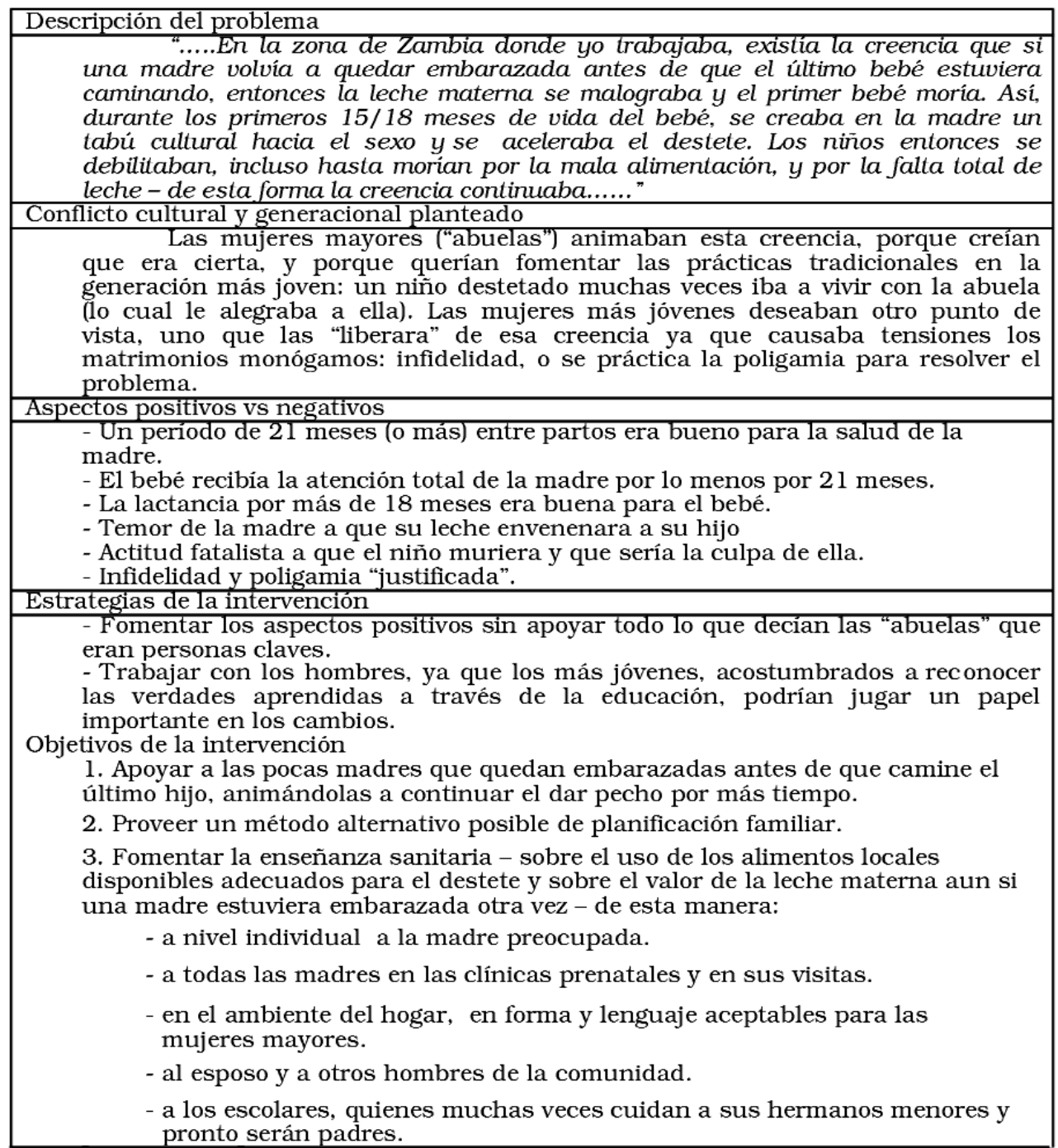

\section{COMPETENCIA CULTURAL EN EL ÁMBITO COMUNITARIO}

En el nivel comunitario la competencia cultural se sustenta igualmente en una efectiva valoración de la diversidad humana desde la que se promueva una convi- vencia pacífica y creativa entre los distintos grupos sociales que conforman un determinado territorio (barrio, pueblo, comunidad de vecinos, etc.). Valorar la diversidad significa que las instituciones y los miembros de la comunidad conocen los beneficios de sus diferencias y simili- 
tudes, y trabajan intencionadamente por construir relaciones sostenibles entre personas e instituciones de diversa pertenencia. Una comunidad que valora la diversidad asegura que sus instituciones proveen igual tratamiento y acceso a recursos y decisiones a todos sus miembros sin tener en cuenta la etnia, la orientación sexual, las creencias religiosas, etc.

La investigación en el área ha constatado que en el ámbito comunitario los programas dirigidos a la mejora de las relaciones intergrupales (inmigrantes vs autóctonos; adultos vs jóvenes, etc.) son un buen referente para la implementación de intervenciones sociales. Estos programas se rigen por una serie de principios estratégicos basados en gran medida en los resultados obtenidos en las investigaciones sobre la teoría del contacto, ya referenciada anteriormente. De acuerdo con la Association for the Estudy and Development of Community (AEDC, 2002), estos principios son:

1. Los grupos implicados deben identificar un problema común y ponerse a trabajar cooperativa y democráticamente, definiendo metas y las estrategias operativas para alcanzarlas. Estas metas deben estar bien definidas y ser suficientemente atractivas y alcanzables; se debe especificar claramente como contribuye cada grupo y resaltar que no podrán ser logradas sin la participación de todos ellos. De acuerdo con la teoria, se espera que los limites intergrupales desaparezcan los individuos se vean a sí mismos como miembros de un único grupo: la comunidad (Brewer, 1996; Worchel y Norvell, 1980; Sherif, 1966).

2. Es importante que los individuos y/o las organizaciones que representan a los distintos grupos de la comunidad estén en idénticas condiciones de poder, respeto e importancia (Hewtston y Brown, 1986).
3. Deben diseñarse actividades comunitarias que le den la oportunidad a los miembros de los distintos grupos a conocerse como individuos.

4. Deben diseñarse actividades en la que, a través de la interacción, los individuos de los distintos grupos identifiquen lo que tienen en común y reconozcan los elementos positivos de los otros grupos: cultura, lenguaje, etc.

5. Los conflictos deben ser identificados, respetados y gestionados de tal forma que mejores las relaciones intergrupales.

6. Se deben festejar las actividades realizadas conjuntamente que culminen con éxito, ya que no sólo mejoran las comunidades en las que viven los grupos, sino también fortalecen sus relaciones.

7. Las situaciones de interacción que se mantienen en el tiempo y cuentan con el apoyo institucional fortalecen las relaciones intergrupales

8. Para ser efectivas, las relaciones entre los distintos grupos de una comunidad deben operar en múltiples niveles: individual, relacional e institucional.

En España, como consecuencia de la inmigración, está incrementándose la diversidad cultural en la composición demográfica de muchos municipios y barrios. Aunque no necesariamente, esta nueva situación tiene que generar un aumento de los problemas vecinales entre los diferentes grupos (población autóctona vs inmigrante, por ejemplo), se dan las circunstancias que justifican intervenciones sociales encaminadas a la promoción de una convivencia en paz y la prevención de conflictos comunitarios. La experiencia que se describe en la Tabla 5, puede ser un buen ejemplo de este tipo de intervención comunitaria. 
Tabla 5. La diversidad avanza en Brodway (AEDC 2002)

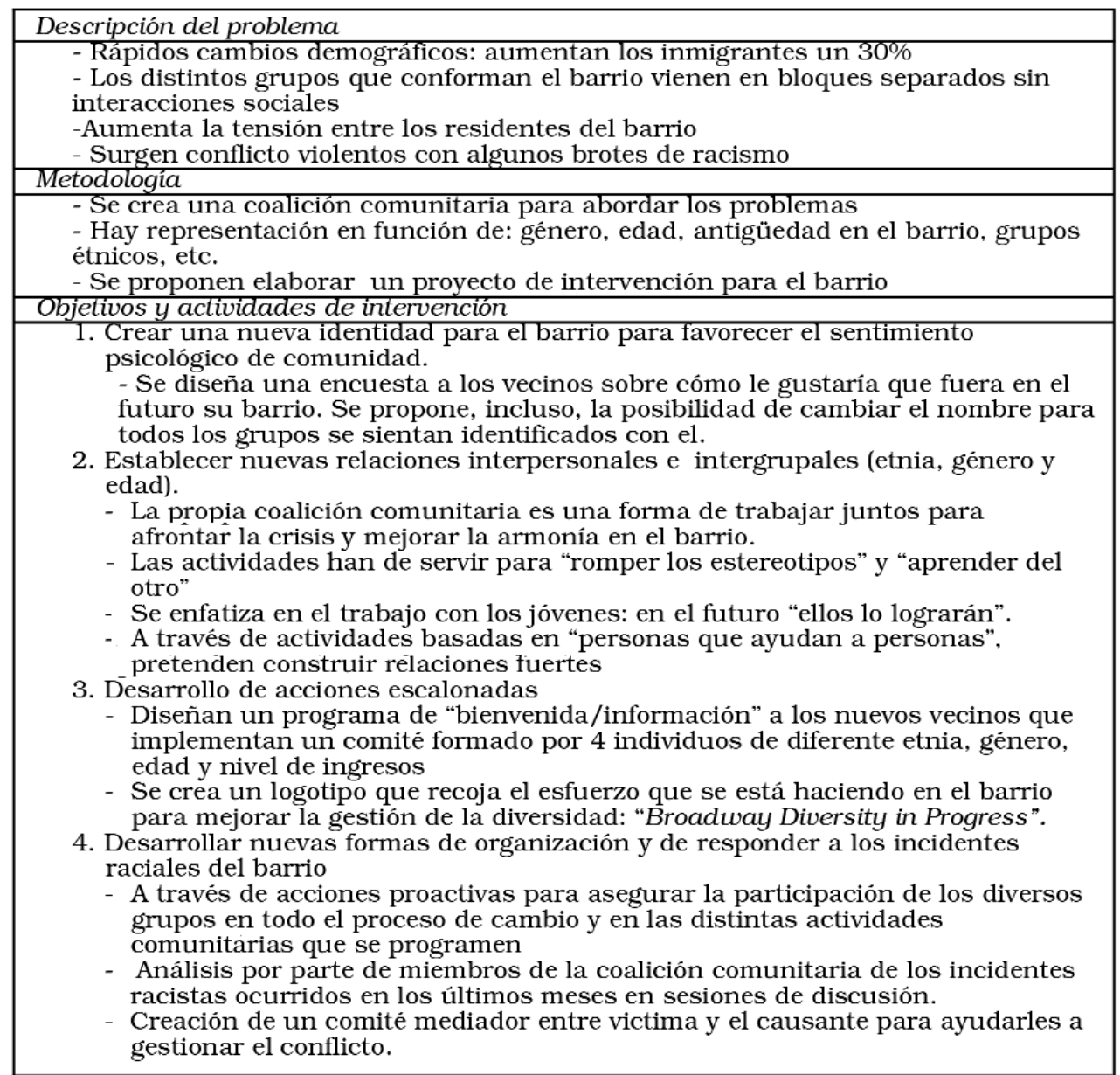

\section{A MODO DE CONCLUSIÓN}

De acuerdo con la actual dinámica en la composición de nuestras poblaciones y a tenor de lo expuesto en las páginas anteriores, el considerar la dimensión cultural en las intervenciones sociales (en todos sus niveles, ámbitos y dimensiones), es uno de los principales retos que tiene planteado el área para los próximos años. Desde esta perspectiva ecológico-cultural se comprenden mejor los problemas de los grupos minoritarios ya que, por una parte, se entiende al individuo dentro de su contexto familiar y a las familias dentro de su contexto cultural. Por otra, se enfatiza la relación de opresión que ejerce la cultura dominante sobre los grupos minoritarios en relación al acceso a los recursos comunitarios, por lo que las intervenciones deben dirigirse a promover relaciones más igualitarias entre todos los grupos (culturales) que componen un determinado contexto comunitario. 
En la práctica, estas dos premisas implican que tanto los servicios asistenciales como los profesionales que los atienden deben actualizar su configuración o formación teórico-practica hasta adquirir una adecuada competencia cultural. Es decir: (1) conformar servicios de atención sensibles a la diversidad cultural; (2) adquirir un grado óptimo de conciencia acerca de la influencia que ejerce nuestro propio background cultural en el desempeño profesional; (3) adquirir conocimientos institucionales y profesionales sobre aspectos culturales de los usuarios (o grupos diana); (3) capacitarse para distinguir entre cultura y patologia (o problema), y (4) adquirir habilidades para integrar esas tres dimensiones en el diseño e implementación de sus intervenciones.

\section{BIBLIOGRAFÍA}

AEDC (2002). The valuing Diversity Project. Final Report.

APA (2003). Guidelines on Multicultural Education, Training, Reasearch, Practoces and Organizational Changes for Psychologists. American Psychologist, 58, 377-402.

Asch, S. (1946). Forming impressions of personality. Journal of Abnormal and Social Psycholo gy, 59, 177-181.

Bernal, G., y Sáez-Santiago, E. (2006). Culturally centered psychosocial interventions. Journal of Community Psychology, 34, 121-131.

Brewer, M.B. (1996). When contact is not enough: Social identity and intergroup cooperation. International Journal Intercultural Relations, 20(3/4), 291-303.

Campinha-Bacote, J. (1998). The process of cultural competence in the delivery of Healthcare Services. Cincinnati, OH: Transcultural C.A.R.E. Associate Press.

Campinha-Bacote, J. (2003). Many Faces: Addressing Diversity in Health Care. Online Journal of Issues in Nursing.

Chen, G. M. y Starosta, W. (1996). Intercultural Communication Competence: A synthesis. Communication Yearbook, 19, 353-383.

Cherpitel, Ch. J. (2001). Differences in services utilization between White and Mexican America DUI arrestess. Alcoholism: Clinical and Experimental Research, 25 (1), 122-127.

Cross, T., Bazron, B., Dennis, K., e Isaacs, M. (1989). Towards A Culturally Competent System of Care, Volume I. Washington, DC: Georgetown Uni- versity Child Development Center, CASSP Technical Assistance Center.

Echeverry, J.J. (1997). Treatment barriers: Assesing and accepting professional help. En G. García y M.C. Zea (Eds.), Psychological interventions and research with Latino populations (p.p. 94-124). Boston: Allyn and Bacon.

Fiske, S. T. (1998). Stereotyping, prejudice, and discrimination. In D. T. Gilbert \& S. T. Fiske (Eds.), The handbook of social psychology, Vol. 2 (4th ed., pp. 357-411). New York: McGraw-Hill.

Hayes, M. (1991). Repport of State Health Officer Washinton. Washinton.

Leinimger, M. (1978). Transcultural nursing. Concepts, theories, research and practice. New York: John Wiley \& Son.

Marin, G. y Marin, B.V. (1991). Research with hispanics populations. Newbury Park, CA. Sage.

Martínez, M.F. y Martínez, J. (2006). Determinantes psicosociales de la utilización de organizaciones comunitarias por inmigrantes. Revista de Psicología General y Aplicada, 59 (3), 433-444.

Michie, S. (2005). Creencias tradicionales y problemas de salud. http://tilz.tearfund.org/.

Nagayama Hall, G. C. (2001). Psychotherapy research with ethnic minorities: Empirical, ethical, and conceptual issues. Journal of Consulting and Clinical Psychology, 69, 502-510.

Nelson, G. \& Prilleltensky, I (2005). The Project of Community Psychology: Issues, Values and Tools for Liberation and Well-being. En G. Nelson \& I. Prilleltensky, I. Community Psychology. In Pur- 
La Competencia Cultural como referente de la Diversidad Humana en la Prestación de Servicios y la Intervención...

suit of Liberation and Well-Being. New York. Palgrave. McMillan.

Pedersen, P. (2000). A handbook for developing multicultural awareness. (3rd ed.). Alexandria, VA: American Counseling Association.

Pedersen, P.D. (2003). Cross-cultural counseling: Developing culture-centered interactions. In G. Bernal, J.E. Trimble, A.K. Burlew, \& F.T.L. Leong (Eds.), Handbook of racial and ethnic minority psychology (pp. 487-503). Thousand Oaks, CA: Sage.

Power, T.J.; Eiraldi, R.B.; Clarke, A. T. \& Mazzuca, L.B. (2005). Improving Mental Health Service Utilization for Children and Adolescents. Social Psychology Quarterly, 20 (2), 187-205.

Purnell, L. y Paulanka, B. (1998). Trancultural health care: A culturally competent approach. Philadelphia: F.A. Davis.

Roberts, R. et al. (1990). Developing Culturally Competent Programs for Families of Children With Special Needs. Washington, D.C.: Georgetown University Child Development Center.

Robinson, C. \& Stalker, K. (1993). Patterns of provision in respite care and the Children Act. British Journal of Social Work, 23(1), 4563.

Saldaña, D. (2001). Cultural Competence. A practical guide for mental health providers. Austin: Hogg Fountation for Mental Healt.

Sherif, M. (1966). Group conflict and co-operation. Their social psychology. Londres: Routledge \& Keagan Paul.

Schofield, J. W. (1986). Causes and consequences of the colorblind perspective. J. F. Dovidio \& S. L. Gaertner (Eds.), Prejudice, discrimination, and racism (pp. 231-253). San Diego, CA: Academic Press.

Sodowsky, G. R., Kuo-Jackson, P., \& Loya, G. (1997). Outcome of training in the philosophy of assessment: Multicultural counseling competencies. In D. B. Pope- Davis \& H. L. K. Coleman (Eds.), Multicultural counseling competencies: Assessment education and training, and supervision. Thousand Oaks, CA: Sage.

Starret, R.A.; Todd, A. \& DeLeon, L. (1989). A comparison of the social service utilization behavior of the Cuban ans Puerto Rican elderly. Hispanic Journal of Behavioral Sciences, 11 (4), 341-353.
Sue, D. W.: Arredondo, P.; y Mcdavis, R.J. (1992). Multicultural counselling competencies and standards: A call to the profession. Journal of Counseling \& Development, 70, 477-486.

Sue, S. y Zane, N. (1987) The rol of culture and culture techniques in psychotherapy: A critique and reformulation, American Psychologist, 42, 37-45.

Sue, D.W.; Ivey, A.F. y Pedersen, P.B. (1996). A theory of multicultural counselling and therapy. San Francisco: Brooks/Cole Publishing.

Sue, S. (2006). Cultural competency: from philosophy to research and practice. Journal of Community Psychology, 34 (2), 237-245.

Sutton, M. (2000). Improving Patient Care. Family Practice Management, 7 (9).

Tajfel, H., \& Turner, J. C. (1986). The social identity theory of intergroup behavior. In S. Worchel \& W. G. Austin (Eds.), Psychology of intergroup relations (pp. 7-24). Chicago: Nelson-Hall.

Trickett, E. J., Watts, R. J., y Birman, D. (1994). Toward an overarching framework for diversity. In E. J. Trickett, Watts, R. J.,y y Birman, D. (Eds.), Human diversity: Perspectives and people in context (pp. 7-26). San Francisco: Jossey-Bass.

Trimble, J. E., Stevenson, M. R., Worell, J. P., et al. (2004). Toward an inclusive psychology: Infusing the Introductory Psychology textbook with diversity content. Washington, DC: American Psychological Association.

Worchel, S. y Norvell, N. (1980). Effect of perceived enviromental conditions during cooperation on intergroup attaction. Journal of Personality and Social Psychology, 18 (5), 764-772.

Vázquez, O. (2002). Competencia intercultural e intervención social. Qué formación para el desarrollo de la acción intercultural. Ponencia al II Congreso nacional sobre Inmigración Extranjera en España.

Wolsko, C., Park, B., Judd, C. M., \& Wittenbrink, B. (2000). Framing interethnic ideology: Effects of multicultural and color-blind perspectives on judgments of groups and individuals. Journal of Personality \& Social Psychology, 78, 635-654.

Zhang, A. Snowden, L.R. \& Sue, S. (1998). Differences between Asian and Whaite American'Help Seeking and Utilization Patterns in the Los Angeles area. Jorunal of Community Psychology, 26 (4), 317-326. 